\title{
Analysis of Music Signal Compression with Compressive Sensing
}

\author{
Urvashi P Shukla \\ PG Student, EC \\ SCET, Surat \\ INDIA
}

\author{
Niteen B Patel \\ Professor, EC \\ SCET, Surat \\ INDIA
}

\author{
Amit M Joshi \\ Research Scholar, EC \\ SVNIT, Surat \\ INDIA
}

\begin{abstract}
Music signal processing has matured over the years. Most of the techniques applied to music signal were originally developed for speech signal; the results observed have been quite satisfactory. In music domain, there are lot many challenges faced with respect to the storage and transmission of large data base. In this paper, we try to address the above stated problems with help of growing compressive sensing field. The aim is to extract the features of the signal with aid of the basis which will in turn reduce the number of samples to be stored or transmitted. Also, our goal is to successfully demonstrate the recovery of the signal without hampering the inner characteristics and the melody of the signal.
\end{abstract}

\section{General Terms}

Compressive sensing, Algorithm, Simulation

\section{Keywords}

Basis matrices, Discrete Fourier Transform, Measurement matrix, Music signal, Recovery algorithm.

\section{INTRODUCTION}

Music is a ubiquitous and vital part of the many lives worldwide. Musical creations and performances are among the most complex and intricate of our cultural artifacts, and the emotional power of music can touch us in surprising and profound ways [1].Music has quiet large span range of forms and styles like simple, unaccompanied folk songs, orchestras and other large ensembles, to a minutely constructed piece of electronic music resulting from months of work in the studio. With a great amount of diversity in the culture various notions are present for music signal. One thing that exists common among them is scale which can be vaguely defined as a set of pitches that repeat itself at a regular interval of time.

These signals are meant for the pleasure of human listeners, and thus its features reflect specific aspects of human auditory perception. In particular, humans perceive two signals whose fundamental frequencies fall in a ratio 2:1 (an octave) as highly similar [2] (sometimes known as "octave equivalence"). Contemporary western music is rested on the "equal tempered" scale, that follows the mathematical coincidence whereby allowing the octave to be divided into twelve equal steps on a logarithmic axis while still preserving intervals corresponding to the most pleasant note combinations. These twelve step also known as the "white notes" on a piano, denoted by C, D, E, F, G, A, B. The lowest note on a piano is A0 $(27.5 \mathrm{~Hz})$, the highest note is C8 $(4186$ $\mathrm{Hz})$, and middle $\mathrm{C}(262 \mathrm{~Hz})$ is $\mathrm{C} 4$ [3].
Now if the signal is composed in accordance of the above method we could reside on concept of fundamental frequency or F0, which are an essential descriptor of harmonic sound signals such as speech and music. Single-F0 estimation algorithms assume that there is at most one harmonic source of which the F0 is to be extracted easily [4]. Music signal is now just limited to the pleasure objective. There many area where its inherit part of the things. Music pieces are taken for authentication purpose[5].Also with the era of digitalization the craze for video game has also increased the use of background music is also there[6].

This paper is to bridge the gap between the sparse representation and its effective utilization. Lots of work has been done for obtaining the sparse representation as it has due advantage the large amount of data turns to be non zero. This reduces the burden on the transmitting end and also the storage requirement. With the single-F0 estimation the presence of harmonic is detected .This can be done with help Fourier Frequency Transform (FFT) or Discrete Fourier Transform (DFT) for discrete data. The single-F0 estimation algorithms have developed. Its applications towards music signals are somehow limited because most music signals contain several concurrent harmonic. Processing of music can also be done in a symbolic framework; most commonly applied is the musical instrument digital interface (MIDI) as the input format. This kind of format exhibits several advantages over audio, since it is based on a considerably reduced amount of data, while incorporating much higherlevel information in the form of note events and orchestration. However, the main limitation is that it loses some fine information available in audio signals such as frequency, amplitude modulations and spectral envelopes, which may be valuable for other tasks.

Next is a mid-level representation based approach where the signal is decomposed into a small number of sound atoms or molecules bearing explicit musical instrument labels. Each atom is a sum of windowed harmonic sinusoidal partials whose relative amplitudes are specific to one instrument. The standard basis is also one of the most common approaches. Where, discrete wavelet transform or a wavelet packet is analyzed. The sparseness is a measure of how fast the DWT coefficients decay, and we are interested in obtaining a representation where the energy of the signal is concentrated in a few of the DWT coefficients. [7]

Organization of the paper has been done in the following manner. It begins with the introduction in section I where a brief overview of the music and mainly concentrating on the western music scale as the signal opted for the analysis belongs to that class. This is followed by the work done to obtain the sparse representation of the music signal. Section II 
has brief overview of Compressive Sensing theory which highlights the important charters ices that one must ponder on to obtained a satisfactory recovery. In section III, the algorithm for our approach is presented. Finally, the approach is thoroughly analyzed and discussed in section IV which is followed by conclusion in section $\mathrm{V}$.

\section{COMPRESSIVE SENSING THOERY}

Compressive Sensing (CS) is a basic mechanism based on the computation of a few non-adaptive linear measurements and still retains the necessary information about $\mathrm{x}$.
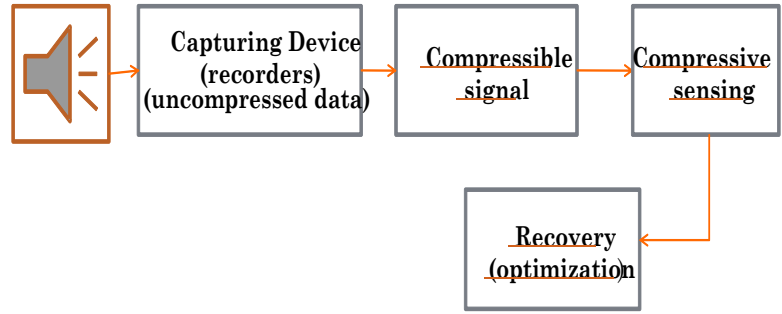

Fig 1: Block diagram for CS system

The above block diagram Fig.1 depicts the whole CS system. To begin with data could be either recorded or generated in some way. Next block is for compressible signal .All the natural signals are not inherit spares but we need to exploit it in order to obtain the one. Sparsity of the signal is defined where number of elements are far less compared to the original sample length. This leads to discard large amount of information without much perpetual loss of information. Let that be known as some domain $\psi$. Where, $\psi$ is called an $\mathrm{N} \times \mathrm{N}$ basis matrix. Here $\mathrm{N}$ is number of the samples in the original signal. With help of this matrix, the signal becomes K sparse.

Then is the compressive sensing block, the measurements done here are represented by a vector

$\mathrm{y}=\Phi \cdot \psi \cdot \mathrm{x}$

Where, $\mathrm{y}$ has dimension $\mathrm{n}<\mathrm{N}, \Phi$ is an $\mathrm{n} \times \mathrm{N}$ measurement matrix (called a CS matrix) and $\mathrm{n}$ are the number of measurements. This leads in dimensional reduction. The measurements $x^{\prime}$ are obtained by employing $\Delta$ referred as the decoder to $\mathrm{y}$ in order to obtain the approximation of $\mathrm{x}$ as given below

$$
x^{\prime}=\text { y. } \Delta(\Phi x)
$$

We need to analyze the role played by each of constitutes of the system in order to appreciate the efforts put on by the CS system. We begin with the sparsity, which pertains to the signal of interest, and incoherence, to the sensing modality through which recovery is assured in CS [8]. The coherence is the measure of the largest correlation between any two elements of $\Phi$ and $\psi$. If $\Phi$ and $\psi$ contain correlated elements, the coherence is large otherwise a small value. For example, pair of spikes and sinusoids is maximally incoherent. We look for minimum value which yields maximum incoherence between two elements in CS.

The next constitute the sensing matrix is non-adaptive. This means that rows of $\Phi$ are fixed in advance and are not depended on the acquired measurements. In order to overcome inaccuracies of the measurements due to the noise at the sensing stage and that due to the mismatches between the CS matrix used during recovery $\Phi$ and during acquisition. Under the condition of above error sources, it is no longer possible to guarantee the uniqueness. It is desirable for the measurement process to be tolerant to both types of error. This behavior is achieved through the restricted isometric property (RIP) [9].

A matrix $\Phi$ has the $(\mathrm{K}, \delta)$ restricted isometric property $((\mathrm{K}, \delta)$ -RIP) if, for all $\mathrm{x} \in \Sigma_{\mathrm{k}}$

$$
(1-\delta)|x| \leq|\phi x| \leq(1+\delta)|x|
$$

Here, $\mathrm{K}$ is the number of non - zero elements in the signal and $\delta$ are the RIP constant. It is a difficult task in practical scenario to construct matrices that incorporate the property of RIP. The limitation is overcome with the help of random matrices like Gaussian and Bernoulli $[12,13]$.

\section{MUSIC CS ALGORITHM}

The normal procedure for any signal remains the same. In the following sections briefly each of the part of the CS process is taken up individually, we begin with measurement model.

\subsection{Measurement model}

As per the theory approach, we can observe that measurement matrix plays a significant role in CS. With the help of it there is dimension reduction. And then the data either could be stored or send over the channel. In our experiment, there are two measurement matrices utilized to conclude on the important role played by them. The first one is random Gaussian matrix generated with help of randn, inbuilt function in Matlab. The mean is taken zero and variance is $1 / \mathrm{K}$ where $\mathrm{K}$ are the number of measurement per frame and the other is Bernoulli where randomly there is permutation of 1's and 0's in the span of $\mathrm{N} \times \mathrm{N}$ matrix. The basis opted is Discrete Fourier Transform (DFT).It is constructed with help of dftmtx function in Matlab for dimension equal to the frame length. The song is constructed out of sinusoids of varying duration and frequency. Thus, sparseness is achieved as nearly two spikes per frame are present instead of huge amount of discrete data obtained by reading the wave file whole file at 8 $\mathrm{KHz}$.

\subsection{Algorithm}

The whole algorithm is presented below. Recovery of the data is done with L1 magic.

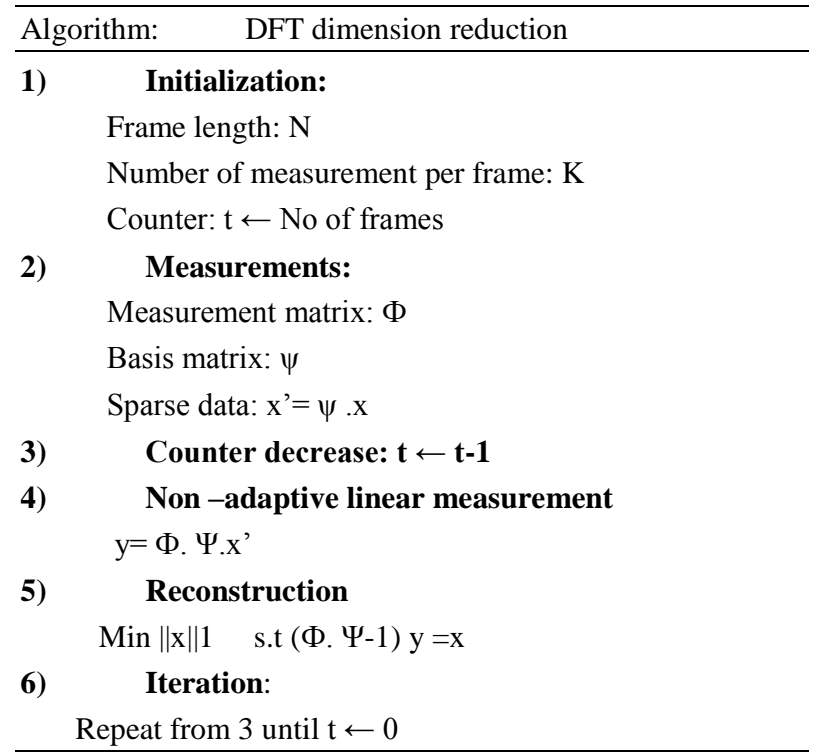


This is basis pursuit (BP) approach where we have taken the case for Min-L1 with equality constraints. Reason for taking $\mathrm{BP}$ is that we have incorporated random measurement models; the method has strongest known guarantees of sparse recovery in presence of random approach $[10,11]$.

\subsection{Performance Matrix}

Total two performance metrics are presented here, to quantify the compression techniques. Aim is to provide with comprehensive information about the compressive sensing with help of which the success ratio can be quantified. The first is Root Mean Square (RMS) error which is calculated as norm2 difference between the original and recovered data. Second, Cross-Correlation (CC) is maximum amount of relation between transmitted and received data. Thirdly, the time required for the reconstruction (RT) of a 1000 sample per frame is also presented. Finally, the compression ratio (CR) is defined as ratio of $\mathrm{K} / \mathrm{N}$ where $\mathrm{K}$ are the number of measurement taken for a frame and $\mathrm{N}$ are the number of samples present per frame.

\section{SIMULATION}

The experiment is conducted on a music piece generated synthetically on Matlab for $49 \mathrm{sec}$ duration. It's the piano based jingle by Ryan McGee in his work Simple Music on the same platform. The sampling rate has been modified to 8 $\mathrm{KHz}$. The whole song is divided into frame each of half a second for faster processing. This test is conducted on i7 Intel Core Processor Clock frequency at $2.65 \mathrm{GHz}$.

In Fig.2 DFT applied on the signal is demonstrated. We could see that most of the terms are zero. So the basis chosen for this analysis is satisfactory to a greater extend.

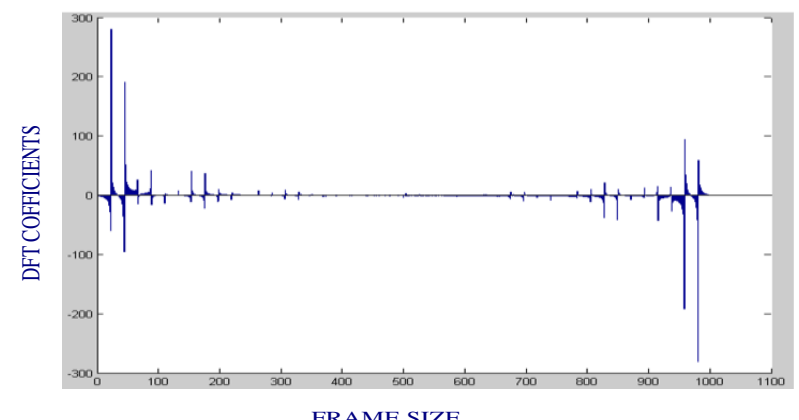

Fig 2: DFT of the signal

Table 1. Detailed performance of music signal for Gaussian measurement matrix

\begin{tabular}{|l|l|l|l|l|l|}
\hline $\boldsymbol{\Psi}$ & $\boldsymbol{\Phi}$ & $\mathbf{C R}$ & $\mathbf{C C}$ & MSE (v) & RT(sec) \\
\hline \multirow{3}{*}{ DFT } & \multirow{3}{*}{ GM } & 0.99 & 4.17 & 0.0019 & 939.33 \\
\cline { 3 - 6 } & & 0.89 & 3.72 & 0.0182 & 961.30 \\
\cline { 3 - 6 } & & 0.79 & 3.37 & 0.6986 & 605.62 \\
\hline
\end{tabular}

Table 1 encapsulates the detailed performance of the applied music signal for Gaussian measurement matrix. The compression of data at the time of data arrival reduces the storage size. With the decrease in CR, the correlation and mean square error have increased. The conclusion to be drawn out is that the reconstruction time is independent of the number of samples.

In Table 2, same analysis is taken up now for the Bernoulli matrix.

\begin{tabular}{|l|l|l|l|l|l|}
\hline $\boldsymbol{\Psi}$ & $\boldsymbol{\Phi}$ & $\mathbf{C R}$ & $\mathbf{C C}$ & MSE (v) & RT(sec) \\
\hline \multirow{3}{*}{ DFT } & \multirow{3}{*}{ BER } & 0.99 & 4.173 & 0.5333 & 956.18 \\
\cline { 3 - 6 } & & 0.89 & 4.176 & 0.6955 & 1007.36 \\
\cline { 3 - 6 } & & 0.79 & 4.174 & 0.7154 & 768.57 \\
\hline
\end{tabular}

Table 2. Detailed performance of music signal for Bernoulli measurement matrix
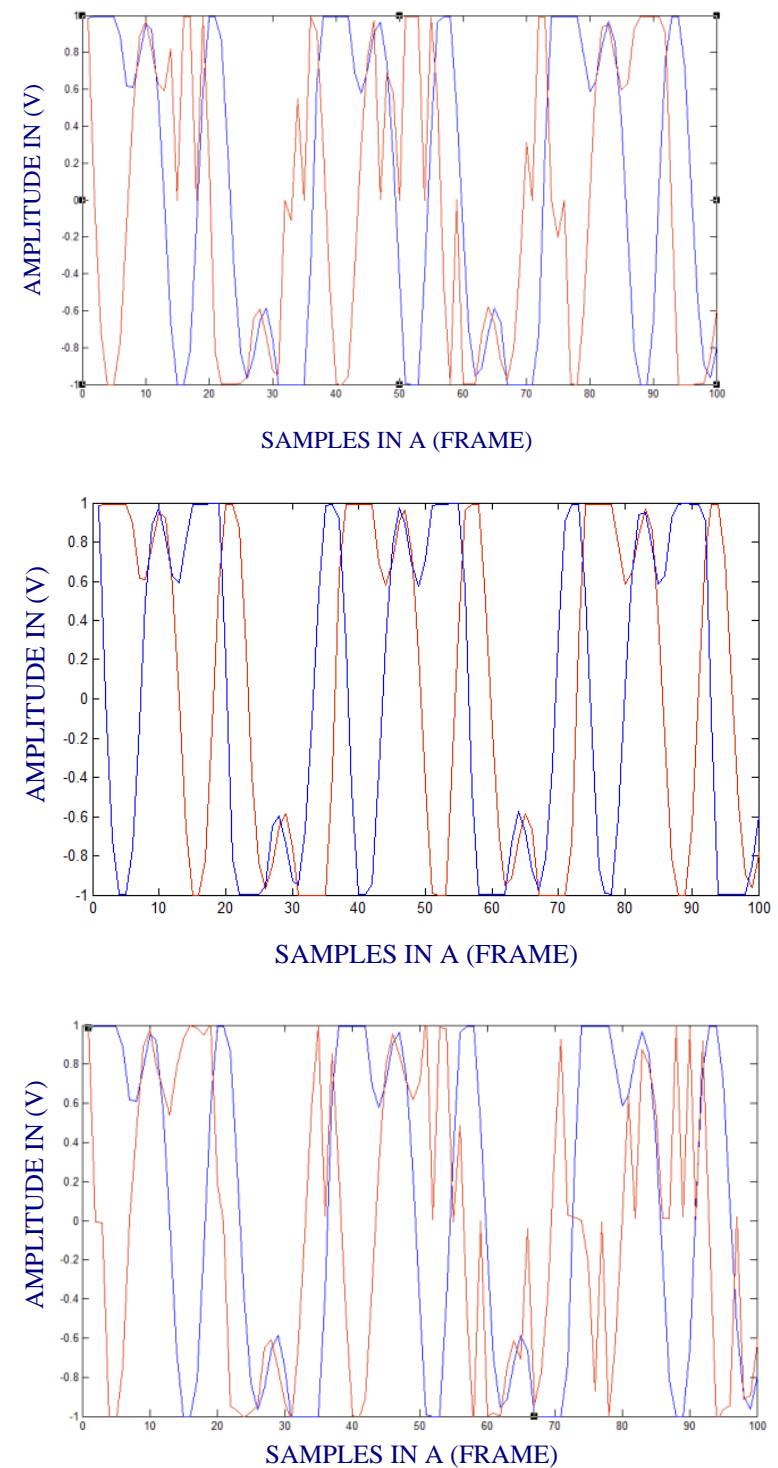

Fig 3: The time domain representation of the original and reconstructed signal for each CR (GM) 

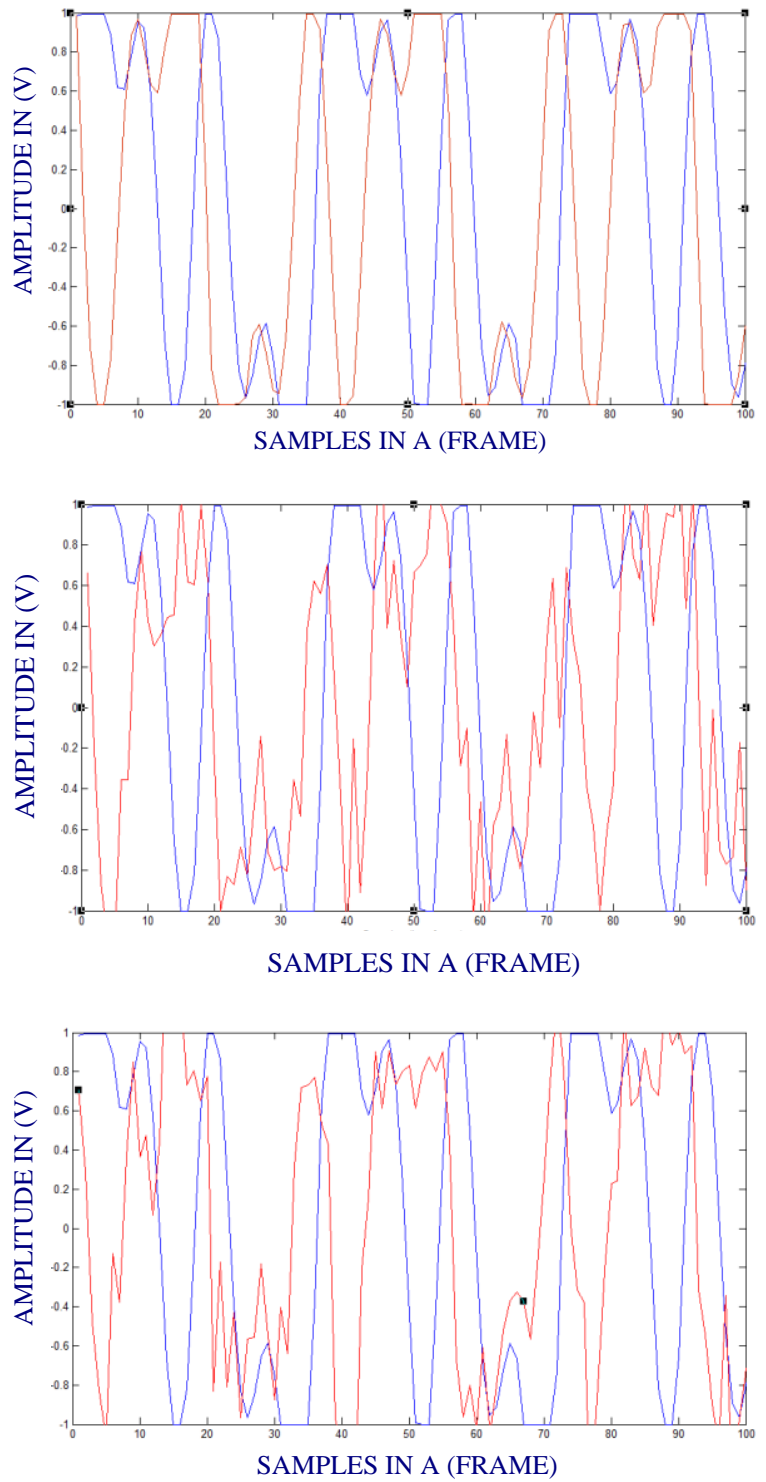

Fig 4: The time domain representation of the original and reconstructed signal for each CR (BER)

The above figures are the snapshots for 100 samples for each different compression ratio. The red are representing the recovered signal and the blue are for the original signal. The constructions are not smooth in nature for Bernoulli as the values are forced to take either of the values from 0's or 1's.

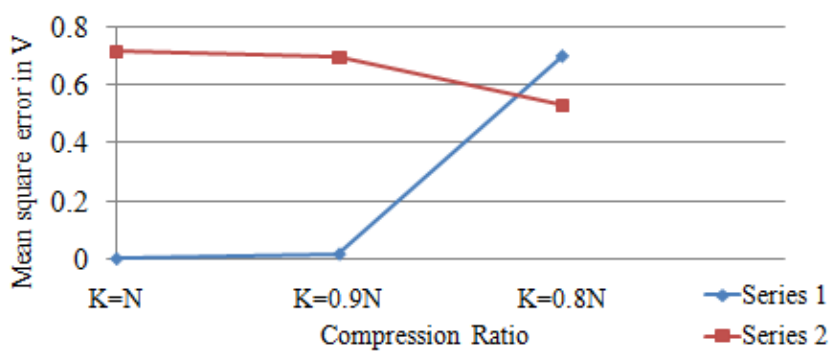

Fig 5: MSE relation with compression ratio

Through the MSE plot it can be inferred that both the approaches are opposite in nature.

\section{CONCLUSION}

This paper is an attempt for applying compressive sensing on music signals which are non stationary in nature. The results depict very rudiment stage as through the results have lighted the scope. Conclusions drawn in this are that with the decrease in the number of samples, the recovery is acceptable and also the required features of the signal are intact. Also, the increase in MSE is due to the introduction of the lag or lead factor in either of the cases. This shortcoming can be overcome with help of synchronization between the transmitting and the receiving side through which MSE will effectively reduce.

\section{ACKNOWLEDGMENTS}

The authors are grateful to all their colleagues and $\mathrm{Mr}$. Prashant P. Shukla for their useful comments and cooperation on many topics related to this work. Also, we would like to thank our organization and staff members for the support and the facilities that have been provided to us.

\section{REFERENCES}

[1] Pertusa, A., \& Inesta, J. M. Efficient methods for joint estimation of multiple fundamental frequencies in music signals. EURASIP Journal on Advances in Signal Processing, 2012(1), pp.1-13.2012.

[2] Meinard Müller, Daniel P. W. Ellis, ,Anssi Klapuri and Gaël Richard, Signal Processing for Music Analysis, IEEE Journal Of Selected Topics In Signal Processing, 5(6), pp.1088-1110, October 2011

[3] Chunghsin Yeh, Axel Roebel and Xavier Rodet,Multiple Fundamental Frequency Estimation and Polyphony Inference of Polyphonic Music Signals, IEEE transactions on audio, speech, and language processing, 18(6), August 2010

[4] Ram K. Nawasalkar, Nilesh M. Shingnapure, Pradeep K. Butey, Extracting Melodic Pattern of 'Mohan Veena' from Polyphonic Audio Signal of North Indian Classical Music, International Journal of Computer Applications ,Recent Trends in Engineering Technology,pp-0975 8887,2013

[5] Naveen Kumar, User Authentication using Musical Password, International Journal of Computer Applications,59(9), pp-0975 - 8887,December 2012

[6] Alamgir Naushad, Tufail Muhammad,Condition Driven Adaptive Music Generation for Computer Games, International Journal of Computer Applications,64( 8),pp-0975 - 8887, February 2013

[7] Zou, Zhenghua, Xinji Liu, and Shu-Tao Xia. "A comparative study of wavelets and adaptively learned dictionary in compressive image sensing." In Signal Processing (ICSP), 2012 IEEE 11th International Conference on, vol. 2, pp. 811-815, 2012.

[8] G. R. G. Baraniuk, Compressive sensing, IEEE Signal Process. Mag., 24(4), pp. 118-120, Jul. 2007.

[9] D. Monajemi, Hatef, Sina Jafarpour, Matan Gavish, David L. Donoho, Sivaram Ambikasaran, Sergio Bacallado, Dinesh Bharadia et al. "Deterministic matrices matching the compressed sensing phase transitions of Gaussian random matrices." Proceedings of 
the National Academy of Sciences 110, no. 4: 11811186,2013 .

[10] E. Rauhut, Holger. "Compressive sensing and structured random matrices." Theoretical foundations and numerical methods for sparse recovery 9 (2010):pp. 192.,2010.

[11] S. Archana, K. A. Narayanankutty, Anand Kumar, Brain Mapping using Compressed Sensing with Graphical Connectivity Maps, International Journal of Computer Applications, pp. 0975 - 8887, 54(11), September 2012
[12] Candès, Oppenheim, J. N., \& Magnasco, M. O. (2013). Human Time-Frequency Acuity Beats the Fourier Uncertainty Principle. Physical Review Letters, 110(4), 044301, 2013.

[13] Sun, G., Zhou, Y., Wang, Z., Dang, W., \& LI, Z. (2012). Sparsity Adaptive Compressive Sampling Matching Pursuit Algorithm Based on Compressive Sensing. Journal of Computational Information Systems, 7(4), pp.2883-2890,2012. 